\title{
Lumped modeling and parameter identification of a hydraulic engine mount
}

\author{
Q. Li ${ }^{1,2, a}$, W.B. Liu ${ }^{1, b}$ A.M. Ji ${ }^{1, c}$ and G. He $e^{1, d}$ \\ 1 Institute of Mechanical and Electrical Engineering, Hohai Univerisity, Changzhou 213022, China \\ 2 Jiangsu Changbao Steel Tube Co., Ltd, Changzhou 213018, China \\ aliqianme@hhu.edy.cn, ${ }^{b}$ weiboliu81@163.com, ${ }^{c}$ jam@ustc.edu.cn, dhegang@hhu.edu.cn
}

\begin{abstract}
Keywords: hydraulic engine mount (HEM); lumped model; parameter identification; vibration isolation character

Abstract. As an important part in vehicle powertrain mounting system, hydraulic engine mounts (HEM) affect the noise, vibration and harshness $(\mathrm{NVH})$ in vehicle greatly. The modeling and parameter identification of the lumped parameters in model has great significance in the design and study of HEM. A lumped model of a hydraulic engine mount based on parameters of the upper and bottom chamber, the rubber spring, the inertia track and the decoupler was established in this study. Then a method to identify the lumped parameters in model was proposed. Finally the results of the dynamic characters by modeling and experiment were compared and analyzed. The analytical methods and conclusions are instructive for the design and tuning of the vibration isolation characteristics of HEMs.
\end{abstract}

\section{Introduction}

Hydraulic engine mount (HEM) or hydraulically damped rubber mount is an vibration isolation part which has upper and bottom chamber in rubber mount, and the hydraulic liquid can flow with the pressure change in the upper chamber and bottom chamber, so HEM can utilize the dissipative function when liquid flow in the upper and bottom chamber to dissipate the vibration energy and obtain the aim to isolate vibration and damping. HEM synthetically utilize the damping characteristic of liquid and the vibration isolation characteristics of rubber mount, it's low stiffness and damping at low frequency, and high stiffness and damping at high frequency can provide a dual damping mode passive vibration isolator to control high-amplitude, low-frequency road-induced vibrations and low-amplitude, high-frequency engine-induced vibrations. HEM can greatly decrease the noise in compartment and increase the comfort in riding to meet increasing customer demand for quieter and smoother riding vehicles [1].

Many researchers have carried through experimental study on the vibration isolation characteristics of HEM. G.kim and R.Singh [2-3] measured the volume stiffness of HEM liquid chamber and the damping in inertia track and established the linear and nonlinear model of HEM basing on the experiment, and simulated the work processes of inertia track and decoupler and the dynamic characteristics of HEM at low frequency. Zhenhua Lu et al. [4] studied on the rule that the pre-load, the vibration amplitude and vibration frequency affect the dynamic characteristics of HDM and its rubber spring by measurement on the static and dynamic mechanical characteristics of inertia track HDM with fixed decoupler, inertia track HDM with floating decoupler and its rubber spring.

In the design and study of HEM, it is important to confirm the dynamic vibration characters of the HEM. A lumped model of a hydraulic engine mount based on parameters of the upper and bottom chamber, the rubber spring, the inertia track and the decoupler was established in this study. A method to identify the lumped parameters in model was also proposed.

This paper focus on experimental study on an inertia track HEM with fixed decoupler illustrated in Fig. 1. In this kind of HEM, the rubber spring supports the engine and acts as a piston, the mount is divided into upper and bottom chamber and liquid can flow in the upper and bottom chamber through the annular inertia track in middle plate. When the upper end of HEM is drove by low-frequency and high-amplitude displacement, the liquid can flow in the upper and bottom chamber through the inertia 
track and HEM dissipates vibration energy by the flow of liquid. When the upper end of HEM is drove by high-frequency and low-amplitude vibration, the dynamic response of liquid in inertia track dwindles and flow ends in high frequency, but the decoupler vibrates along with the drive.

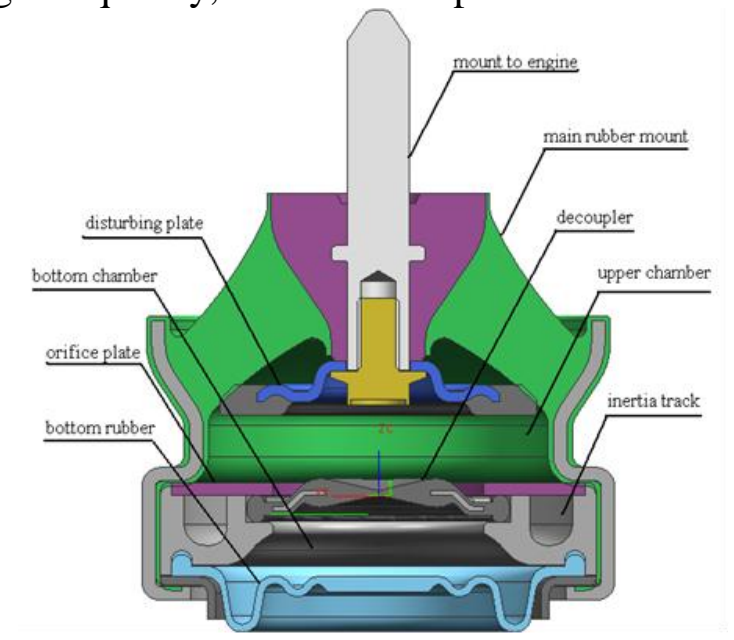

Fig. 1. Cross-section of a HEM

\section{Lumped Model of the HEM}

The principal physical effects are taken to be those associated with the primary rubber including dynamic stiffness and damping, bulge stiffness, piston area and with the inertia track including fluid inertia and damping [3]. The stiffness of the secondary rubber is small enough that it can be ignored for reducing the requisite state dimension by one. The fluid is assumed incompressible for the bulge compliance of the primary rubber is much greater and the fluid inertia and damping in the upper chamber are ignored for inertia and damping in the track is much greater. Considering the HEM in Fig.1 at large amplitude drive, the LP model for this HEM is shown in Fig. 2. The rubber spring is simplified as one degree of freedom (DOF), and its dynamic stiffness and damping property are described by $K_{r}$ and $B_{r}$, respectively. The rubber spring also functions as a piston with an effective area $A_{p}$. Finally, the rubber spring adds volumetric compliance to the model, represented by $C_{l}$. The reciprocal of the $C_{1}$ is defined as the volumetric stiffness of the upper chamber and is represented by $K_{b}$. The bulge stiffness of the upper chamber is defined as

$$
K_{b}=\Delta p_{1} / \Delta v_{1}
$$

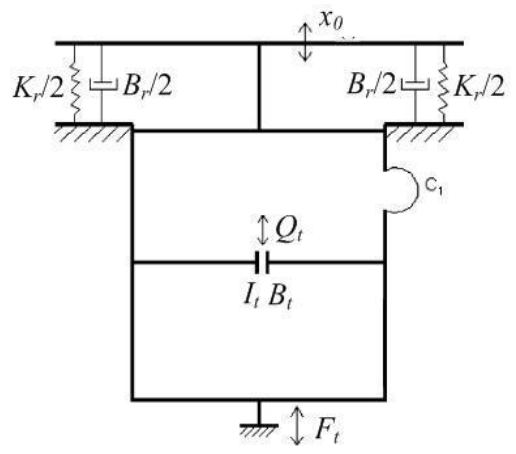

Fig. 2. Lumped model of the HEM

Where $\Delta V_{1}$ is the volume change of the upper chamber which due to the pressure change of the upper chamber, $\Delta P_{1}$. Since the fluid in the chambers is almost incompressible, the volumetric stiffness only depends on the configuration and the Shore hardness of the rubber spring. The fluid in the inertia track is assigned lumped parameters $I_{t}, B_{t}$, which represents the track inertia, and track damping, respectively. The lumped inertia parameter, $I_{t}$, is defined as

$$
I_{t}=m_{t} / a_{t}^{2}=\rho l_{t} / a_{t}
$$


Where $\rho$ is the density of the fluid in chambers, $m_{t}$ is the fluid mass in the inertia track, $l_{t}$ and $a_{t}$ are length and cross-sectional area of the inertia track, respectively.

State equations and an output equation for the reaction force may be derived from the mechanical equivalent model. The states are shear displacement of the rubber spring $x_{r}$, bulge displacement of the primary rubber $V_{b}$ and volumetric flow in the inertia track $Q_{t}$.

$$
\begin{aligned}
& {\left[\begin{array}{c}
\dot{x}_{r} \\
\dot{V}_{b} \\
\dot{Q}_{t}
\end{array}\right]=\left[\begin{array}{ccc}
0 & 0 & 0 \\
0 & 0 & -1 \\
0 & K_{b} / I_{t} & -B_{t} / I_{t}
\end{array}\right]\left[\begin{array}{c}
x_{r} \\
V_{b} \\
Q_{t}
\end{array}\right]=\left[\begin{array}{c}
1 \\
A_{p} \\
0
\end{array}\right] \dot{x}_{0}} \\
& F=\left[\begin{array}{lll}
K_{r} & A_{p} K_{b} & 0
\end{array}\right]\left[\begin{array}{c}
x_{r} \\
V_{b} \\
Q_{t}
\end{array}\right]+\left[B_{r}\right] \dot{x}_{0}
\end{aligned}
$$

The transfer function associated with the model is

$$
K_{d y n}(s)=\frac{F(s)}{X(s)}=K_{r}+B_{r} s+A_{p}^{2} K_{b} \frac{I_{t} s^{2}+B_{t} s}{I_{t} s^{2}+B_{t} s+K_{b}} .
$$

\section{Methods for Obtaining the Lumped Parameters}

The methods for obtaining the lumped parameters and the exactness of the estimated parameters are critical to the simulated dynamic properties of an HEM. In the following section, the methods for obtaining these parameters are discussed.

Stiffness and Damping of Rubber Springs. Since it is difficult to get the dynamic stiffness of a rubber spring for different excitation amplitudes and frequencies by calculating methods, and the dynamic stiffness of a rubber spring contributes slightly to the dynamic stiffness of an HEM, the rubber spring's dynamic stiffness is usually assumed to be a constant, and is estimated from Eq. 6 .

$$
K_{r}=\lambda K_{s}
$$

Where $\lambda$ is a correction factor that is generally in the range from 1.2 to 1.6 , and $K_{s}$ the static stiffness can be obtained by experiment or finite element analysis method.

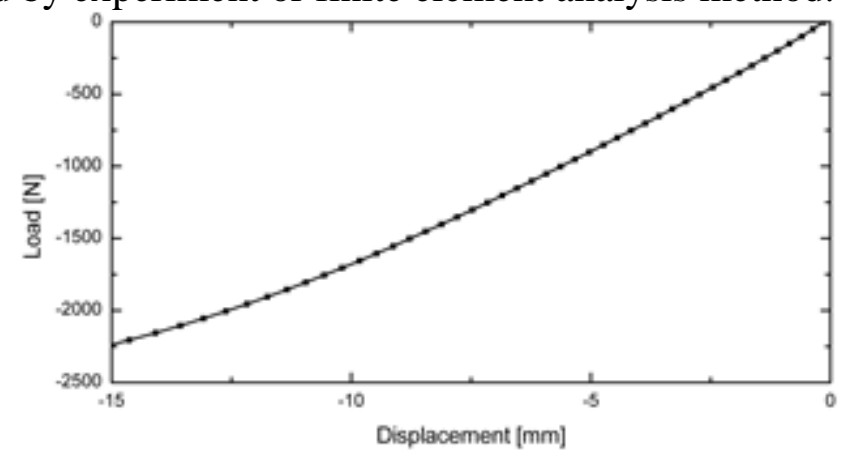

Fig. 3. Compression force versus displacement of the rubber spring

Fig. 3 is the compression force versus displacement curve of the rubber spring by experimental method. The slope ratio of the curve can be seen as static stiffness. The static stiffness of the sample is $151.02 \mathrm{~N} / \mathrm{mm}$. The correction factor is selected as 1.4 in this study. So the dynamic stiffness of the sample is $211.43 \mathrm{~N} / \mathrm{mm}$.

The damping ratios, $\xi_{r}$, of the natural rubber material NR50 is 0.069 in this study. The damping of the spring rubber can be calculated using Eq. 7. 


$$
B_{r}=2 \xi_{r} \sqrt{M K_{r}}
$$

Where $M$ is the equivalent mass of the spring rubber equals $0.25 \mathrm{~kg}$ in this study. The damping of the spring rubber is $3.17 \mathrm{~N} . \mathrm{s} / \mathrm{mm}$.

Effective Piston Area of the Rubber Spring. The rubber spring is deformed under the pre-load of the powertrain, which influences the effective piston area. Experimental data and calculated result show that the area is almost invariable with the pre-displacement of a rubber spring if the pre-displacement is great than $5 \mathrm{~mm}$. In most cases, the pre-displacement of an HEM used in powertrain mounting system is usually larger than $6 \mathrm{~mm}$. So the effective piston area is usually regarded as a constant, is equal to the area of the orifice plate top surface, which is easily measured before the rubber spring is manufactured. The effective piston area of the spring rubber studied in this study is $5.278 \times 10^{-3} \mathrm{~m}^{2}$.

Bulge Stiffness. Since the fluid in the chambers is almost incompressible, the bulge stiffness of the upper chamber is determined mostly by the hardness of the rubber spring. Fig. 4 shows an experimental method to measure the bulge stiffness of the upper chamber. Adjust the pressure in the circuit using the pressure adjuster, record the variable of the liquid altitude and pressure in the circuit. The pressure versus volume variation in the upper chamber was recorded in Fig. 5. The bulge stiffness can be calculated using Eq. 1 as $2.423 \times 10^{9} \mathrm{~N} / \mathrm{m}^{5}$.

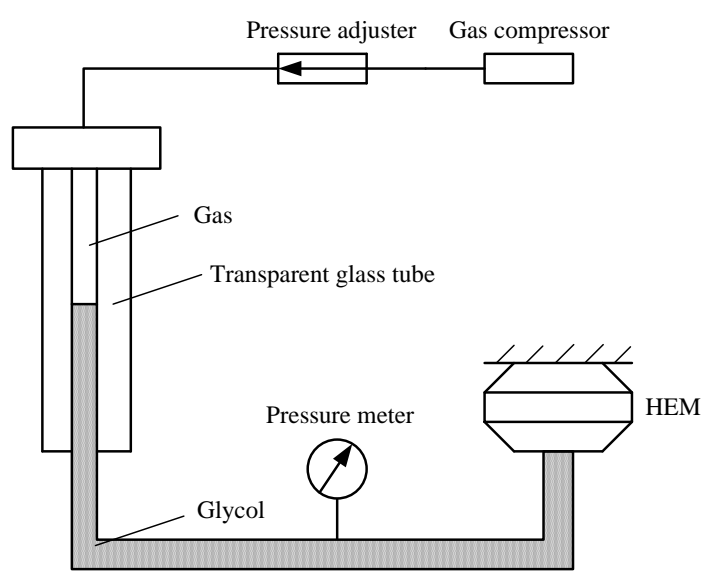

Fig. 4. Experimental rig to measure the bulge stiffness of the upper chamber

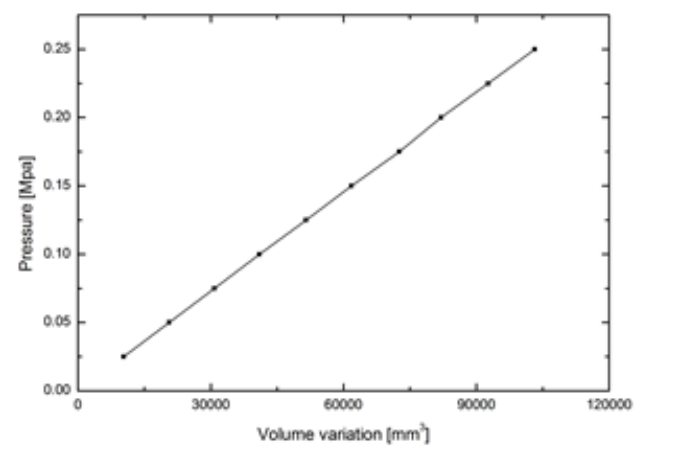

Fig. 5. The pressure versus volume variation in the upper chamber

Lumped Parameters of the Inertia Track. The lumped parameters of an inertia track are the track inertia, $I_{t}$, and the track damping, $B_{t}$. The methods to determine these two parameters include experiment, simulation and empirical equation. Eq. 2 and Eq. 8 are selected to determine track inertia and track damping, respectively.

$$
P_{1}-P_{2}=B_{t} Q_{t}=\frac{128 \mu l_{t}}{\pi d_{t}^{4}} Q_{t}
$$

Where $\mu, l_{t}$ and $d_{t}$ are viscosity of fluid, the length of the inertia and the hydraulic diameter of the inertia track, respectively, and these variables are invariable with excitation amplitudes. The track inertia and track damping of the HEM are $3.227 \times 10^{6} \mathrm{~kg} / \mathrm{m}^{4}$ and $4.007 \times 10^{7} \mathrm{~N} \cdot \mathrm{s} / \mathrm{m}^{5}$. 


\section{Results}

The dynamic characters of the HEM were obtained by experiment and simulation by the model established in this study as illustrated in Fig. 6. It can be seen from the figure the experimental results agree well with the simulated results. The model and the parameter identification method proposed in this study are valid.

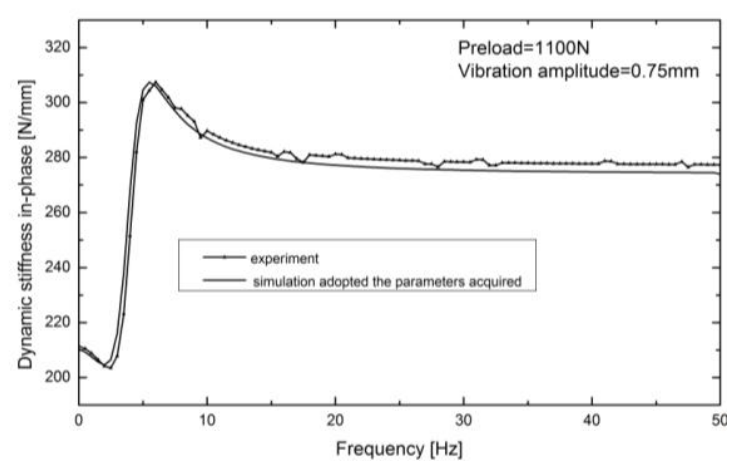

(a) Dynamic stiffness

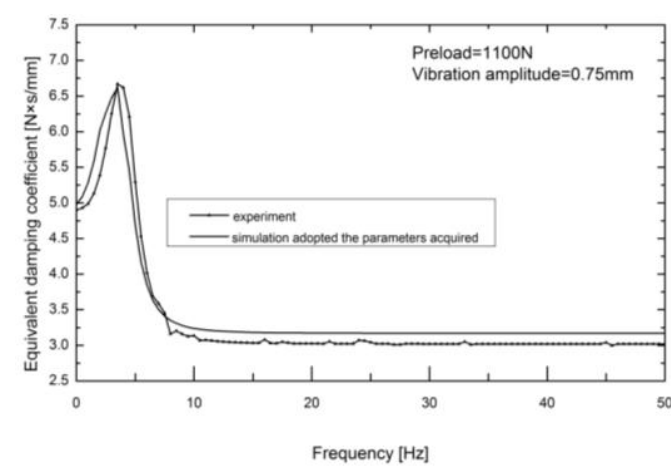

(b) Damping coefficient

Fig. 6. The dynamic characters of the HEM by experiment and simulation

\section{Conclusions}

A lumped model of a hydraulic engine mount based on parameters of the upper and bottom chamber, the rubber spring, the inertia track and the decoupler was established in this study. And the method to identify the lumped parameters in model was also proposed. The dynamic characters of the HEM obtained by experiment agree well with the simulated dynamic characters by the model established in this study. The analytical methods and conclusions are instructive for the design and tuning of the vibration isolation characteristics of HEMs.

\section{Acknowledgements}

This work was financially supported by the Natural Science Foundation of Jiangsu Province of China (Grant No. BK20130237) and the Key Project of Science and Technology of Jiangsu (Grant No. BY2015030-02).

\section{References}

[1] Christopherson, J., Nakhaie Jazar, G.: J SOUND VIB, Vol. 290 (2006)), pp.1040

[2] Singh, R., Kim, G., Ravindra, P.V.: J SOUND VIB, Vol. 158 (1992), pp.219

[3] Kim, G. and Singh, R.: ASME Journal of Dynamic Systems, Measurement and Control. Vol. 115 (1993), pp.482

[4] R. Fan, Z. Lu: J SOUND VIB, Vol. 305 (2007), pp.703 